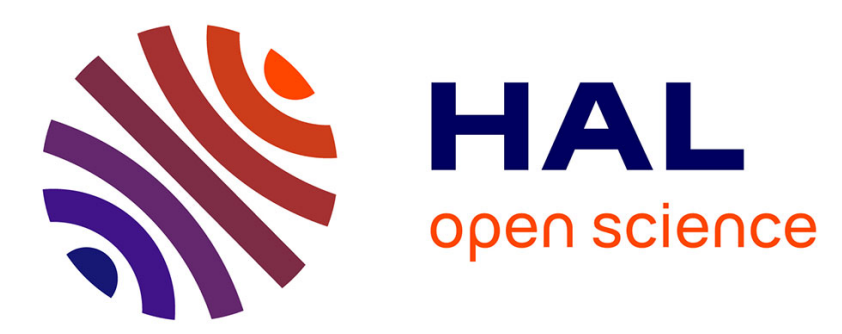

\title{
Retention of Eucalyptol, a Natural Volatile Insecticide, in Delivery Systems Based on Hydroxypropyl- $\beta$-Cyclodextrin and Liposomes
}

Riham Gharib, Jouda Mediouni Ben Jemâa, Catherine Charcosset, Sophie Fourmentin, Helene Greige-Gerges

\section{To cite this version:}

Riham Gharib, Jouda Mediouni Ben Jemâa, Catherine Charcosset, Sophie Fourmentin, Helene GreigeGerges. Retention of Eucalyptol, a Natural Volatile Insecticide, in Delivery Systems Based on Hydroxypropyl- $\beta$-Cyclodextrin and Liposomes. European Journal of Lipid Science and Technology, 2020, 122 (5), pp.1900402. 10.1002/ejlt.201900402 . hal-03033940

\author{
HAL Id: hal-03033940 \\ https://hal.science/hal-03033940
}

Submitted on 1 Dec 2020

HAL is a multi-disciplinary open access archive for the deposit and dissemination of scientific research documents, whether they are published or not. The documents may come from teaching and research institutions in France or abroad, or from public or private research centers.
L'archive ouverte pluridisciplinaire HAL, est destinée au dépôt et à la diffusion de documents scientifiques de niveau recherche, publiés ou non, émanant des établissements d'enseignement et de recherche français ou étrangers, des laboratoires publics ou privés. 


\section{Retention of eucalyptol, a natural volatile insecticide, in delivery systems based on hydroxypropyl-ß-cyclodextrin and liposomes}

Riham Gharib ${ }^{1,2,3}$, Jouda Mediouni Ben Jemâa ${ }^{4}$, Catherine Charcosset, ${ }^{2}$ Sophie Fourmentin $^{3}$, Hélène Greige-Gerges ${ }^{1 *}$

${ }^{1}$ Bioactive Molecules Research Laboratory, Faculty of Sciences, Doctoral School of Sciences and Technologies, Lebanese University, Lebanon.

${ }^{2}$ Laboratoire d'Automatique et de Génie des Procédés, Université Claude Bernard Lyon 1, UMR 5007, CNRS, CPE, 43 bd du 11 Novembre, 691622 Villeurbanne Cedex, France ${ }^{3}$ Unité de Chimie Environnementale et Interactions sur le Vivant (UCEIV), EA 4492 SFR Condorcet FR CNRS 3417, Université du Littoral-Côte d'Opale, 59140 Dunkerque, France.

${ }^{4}$ Laboratory of Biotechnology Applied to Agriculture, National Agricultural Research Institute of Tunisia (INRAT), University of Carthage, Tunisia

*Corresponding author: Hélène Greige-Gerges, Professor, Faculty of Sciences, Section II, Bioactive Molecules Research Laboratory, Lebanese University, B.P. 90656, Jdaidet El-Matn, Lebanon. $\quad$ Tel: $\quad 961-3 \quad 341011 . \quad$ E-mail: greigegeorges@yahoo.com; hgreige@ul.edu.lb

Keywords: conventional liposomes; drug-in-cyclodextrin-in-liposomes; eucalyptol; freeze-drying; hydroxypropyl-ß-cyclodextrin.

Abbreviations: conventional liposome (CL); cyclodextrin (CD); 1,6-diphenylhexatriene (DPH); encapsulation efficiency (EE); eucalyptol (Euc); drug-in-cyclodextrin-inliposome (DCL); hydroxypropyl-ß-cyclodextrin (HP-ß-CD); loading capacity (LC); 
loading rate (LR); multiple head extraction (MHE); transmission electron microscope (TEM). 


\section{Abstract}

2 Eucalyptol (Euc) is a natural monoterpene with insecticide effects. Being highly volatile

3 and sensitive to ambient conditions, its encapsulation would enlarge its application. Euc-

4 loaded conventional liposomes (CL), cyclodextrin/drug inclusion complex and drug-in-

5 cyclodextrin-in-liposomes (DCL) were prepared to protect Euc from degradation, reduce

6 its evaporation and provide its controlled release. The liposomal suspension was freeze-

7 dried using hydroxypropyl-ß-cyclodextrin (HP-ß-CD) as cryoprotectant. The liposomes

8 were characterized before and after freeze-drying. The effect of Euc on the fluidity of

9 liposomal membrane was also examined. A release study of Euc from delivery systems,

10 in powder and reconstituted forms, was performed by multiple head extraction at $60{ }^{\circ} \mathrm{C}$

11 after 6 months of storage at $4{ }^{\circ} \mathrm{C}$. CL and DCL suspensions were homogeneous, showed

12 nanometric vesicles size, spherical shape and negative surface charge before and after

13 freeze-drying. Moreover, HP-ß-CD did not affect the fluidity of liposomes. CL

14 formulations presented a weak encapsulation for Euc. The loading capacity of eucalyptol

15 in DCL was 38 times higher than that in CL formulation. In addition, freeze-dried DCL

16 and HP-ß-CD/Euc inclusion complex showed a higher retention of eucalyptol than CL

17 delivery system.

18 Both carrier systems HP-ß-CD/Euc and Euc-loaded DCL decreased Euc evaporation and 19 improved its retention. 


\section{Introduction}

21 Eucalyptol (Euc) or 1,8-cineole, is a natural monoterpene. It is a major compound of

22 many plant essential oils and is mainly extracted from Eucalyptus globulus and

23 Rosmarinus officinalis essential oils ${ }^{[1]}$.

24 Batish et al. ${ }^{[2]}$ pointed out that 1,8-cineole supports the pesticidal activity of Eucalyptus

25 oils. Similarly, Scriven and Meloan ${ }^{[3]}$ reported that cineole act as a natural repellent

26 against Periplaneta americana. Moreover, Corbet et al., ${ }^{[4]}$ proved that cineole is a

27 mosquito larvicide. Additionally, Euc is also used as a fumigant agent against insect-pest

28 of stored grains ${ }^{[5]}$. Considering the repellent index, it was reported that Eucalyptos

29 kruseana oil showed the highest repellency as compared to other essential oils ${ }^{[6]}$. In

30 human, Euc has been used to treat lung inflammation and respiratory diseases and is

31 found as active principle in a commercial medicinal product "Soledum ${ }^{\circledR}$ Kapseln"

32 contained pure isolated monoterpene 1,8 -cineol ${ }^{[1]}$, and it has been demonstrated that Euc

33 showed neuroprotective effects in an ischemic stroke model ${ }^{[7]}$.

34 To our knowledge there is no data concerning the stability study of eucalyptol under light

35 and at various temperatures. The product is recommended to be stored at $4^{\circ} \mathrm{C}$ and in a

36 dark bottle. Generally, chemical reactions are accelerated with increasing heat. Some

37 essential oil components including eucalyptol are known to easily convert into each other

38 by oxidation, isomerization, cyclization, or dehydrogenation reactions, triggered either

39 enzymatically or chemically ${ }^{[8]}$. The chemical oxidation of Euc has been carried out by

40 strong oxidizing reagents to give oxo compounds (3-oxo-1,8-cineole, 5-oxo-1,8-cineole

41 and 3,5-dioxo-1,8-cineole) ${ }^{[9]}$. The sites of oxidation are the carbon 3 and/or 5 . The

42 encapsulation of Euc can be considered as a potential solution to overcome the 
43 drawbacks related to its physico-chemical properties.

44 Based on the drug-in-cyclodextrin-in-liposomes (DCL) delivery system proposed by

45 McCormack \& Gregoriadis ${ }^{[10]}$, we developed many formulations loading essential oil

46 compounds to protect them from degradation, to achieve their controlled release, and to

47 facilitate their handling through preparation in freeze-dried forms ${ }^{[11,12,13]}$. The DCL

48 system, combining the relative advantages of both carriers, improved the encapsulation of

49 many bioactive compounds such as trans-anethole [11], eugenol [14], estragole [13],

50 quercetin ${ }^{[15]}$, nerolidol ${ }^{[16]}$, and reduced their release compared to conventional liposomes $51[11,13]$.

52 Cyclodextrins (CDs) are cyclic oligosaccharides consisted of glucopyranose units. They

53 are non-hygroscopic substances, homogeneous and crystalline ${ }^{[17]}$. CDs have a form of a

54 truncated cone with an internal hydrophobic cavity and an external hydrophilic surface.

55 This particular structure confers to CDs the ability to form inclusion complexes with a

56 large number of hydrophobic guest molecules ${ }^{[18]}$. Among numerous CDs,

57 hydroxypropyl- $\beta$-cyclodextrin (HP- $\beta-C D)$ was shown to be very effective for Euc

58 encapsulation ${ }^{[18]}$.

59 Liposomes are microscopic vesicles in which an aqueous volume is entirely enclosed by

60 a membrane. They are usually made of natural, biodegradable, non-toxic and non-

61 immunogenic lipid molecules ${ }^{[19]}$. In aqueous media, the phospholipids can slowly

62 become oxidized or hydrolyzed ${ }^{[20]}$ and this could induce liposomes membrane

63 destabilization and leakage of the encapsulated drug. Freeze-drying liposomes increased

64 their shelf-life ${ }^{[21]}$; however during freeze-drying the disruption of liposome membrane

65 may occur leading to vesicles aggregation ${ }^{[20]}$; for that cryoprotecting agents such as HP- 
66 B-CD are generally used to stabilize liposome structures during freeze-drying ${ }^{[12]}$.

67 In this study, ethanol injection method was applied using hydrogenated soybean

68 phospholipid and cholesterol to prepare conventional liposomes (CL) and DCL. These

69 liposomes were characterized for their size, polydispersity index, zeta potential and

70 morphology before and after freeze-drying. To obtain reproducible and efficient

71 insecticidal effects, the homogeneity and the stability of liposome formulations should be

72 ensured. For that, the particle size distribution value of the batches was determined. Zeta

73 potential is a parameter used to evaluate the physical stability of liposomal formulations.

74 It characterizes the particles surface charge and gives an indication about repulsive forces

75 between particles, thus allowing predicting stability of colloidal dispersions ${ }^{[22]}$. The

76 loading rate of Euc was determined after its HPLC analysis. The release of Euc from the

77 inclusion complex, CL and DCL was studied by multiple headspace extraction at $60^{\circ} \mathrm{C}$.

78 Hence, the fluorescence anisotropy of 1,6-diphenylhexatriene (DPH) inserted in the lipid

79 membranes of CLs and DCLs was studied before and after freeze-drying at 25 and $37^{\circ} \mathrm{C}$.

\section{Materials and methods}

\section{$81 \quad 2.1 \quad$ Materials}

82 Hydrogenated phospholipon $90 \mathrm{H}$ (90\% soybean phosphatidylcholine, $4 \%$

83 lysophosphatidylcholine, 2\% triglycerides, $2 \%$ water, $0.5 \%$ ethanol, $1 \%$ iodine) was

84 supplied by Lipoid GmbH (Ludwigshafen, Germany). HP-ß-CD-oral grade (MS=0.85)

85 was obtained from Roquette (Lestrem, France), eugenol, absolute ethanol, cholesterol and

86 methanol-HPLC grade were from Sigma-Aldrich (Germany) and Euc was purchased

87 from Sigma-Aldrich (USA). Water was purified on a Milli-Q system obtained from a

88 Millipore® synergy system (Millipore, Billerica, Massachusetts, USA). 


\section{$89 \quad 2.2$ Preparation of HP-ß-CD/Euc inclusion complex}

90 Excess amount of Euc (7.712 mg) was added to $5 \mathrm{~mL}$ HP-ß-CD solutions $(0,10,25,50$,

91 and $100 \mathrm{mM}$ ). The mixtures were shaken at $25^{\circ} \mathrm{C}$ for $24 \mathrm{~h}$ then filtered through a 0.45

$92 \mu \mathrm{m}$ membrane filter. The HPLC method described below was used to determine the

93 concentrations of Euc in the filtrates.

94 The encapsulation efficiency was calculated using the following equation:

$$
E E_{C D}(\%)=\frac{m E u c_{e x p}}{m E u c_{i}} \times 100(1)
$$

95 where $\mathrm{m} \mathrm{Euc}_{\exp }$ is the mass of Euc experimentally determined by HPLC in the inclusion

96 complex solution and $\mathrm{m} \mathrm{Euc}_{\mathrm{i}}$ is the mass of Euc initially used to prepare the inclusion 97 complex.

$98 \quad 2.3$ Preparation of HP-B-CD/Euc inclusion complex for DCL preparations

99 HP-ß-CD (50 mM, $74 \mathrm{mg} / \mathrm{mL})$ was dissolved in ultrapure water and the required amount 100 of Euc was added to obtain HP-ß-CD:Euc molar ratio of 5:1. HP-ß-CD/Euc inclusion 101 complex was prepared as described above and the solution was used for DCL 102 preparations.

\section{$103 \quad 2.4$ Preparation of liposomes by ethanol injection method}

104 The liposomes were prepared by the ethanol injection method using Phospholipon 90H

$105(10 \mathrm{mg} / \mathrm{mL})$ and cholesterol $(5 \mathrm{mg} / \mathrm{mL})$ according to the protocol described previously

$106{ }^{[11]}$. Different formulations were prepared: 1) blank-CL; 2) blank-DCL, where HP-B-CD

107 was dissolved in the aqueous phase at a concentration of $50 \mathrm{mM}$; 3) Euc-loaded

108 liposomes (Euc-CL), where Euc was added in the organic phase at a concentration of 2.5

$109 \mathrm{mg} / \mathrm{mL}$; 4) HP-ß-CD/Euc inclusion complex-loaded liposomes (Euc-DCL), where HP-ß3-

$110 \mathrm{CD} /$ Euc inclusion complex obtained at molar ratio of 5:1 was used as an aqueous phase. 
111 Each batch was prepared in triplicate and underwent characterization as described below.

\section{$112 \quad 2.5 \quad$ Freeze-drying}

113 Freshly prepared liposomes $(5 \mathrm{~mL})$ were freeze-dried according to the protocol described

114 in our previous study ${ }^{[12]}$, where HP-B-CD was used as a cryoprotector agent at

115 concentrations of 25 and $50 \mathrm{mM}$ for $\mathrm{CL}$ and $50 \mathrm{mM}$ for DCL formulations. The obtained

116 powders were then reconstituted with ultra-pure water to the original volume $(5 \mathrm{~mL})$

117 prior to characterization and further analysis.

118 HP-B-CD/Euc inclusion complex was also freeze-dried and studied for its ability to retain

119 Euc as described below.

\section{$120 \quad \mathbf{2 . 6}$ Liposome characterization}

\section{$121 \quad$ 2.6.1 Dynamic Light Scattering Analysis}

122 Malvern Zetasizer Nanoseries (Zetasizer Nano ZS; Malvern Instruments Ltd, France) was

123 used to determine the mean size, polydispersity index and zeta potential values of 124 liposome batches ${ }^{[12]}$.

\section{$125 \quad$ 2.6.2 Morphological characterization by transmission electron microscopy}

126 Blank-DCL and Euc-DCL before and after freeze-drying were imaged with transmission 127 electron microscope (TEM) (CM 120; Philips, Eindhoven, Netherlands) according to the 128 protocol described by Gharib et al ${ }^{[11]}$.

\section{$129 \quad$ 2.6.3 Determination of encapsulation efficiency and loading rate of Euc}

130 The EE of Euc into liposomes was calculated after determination of the concentrations of

131 free and total Euc present in the liposome suspensions by HPLC. Aliquots were removed

132 from the liposomal suspension to determine the total Euc concentration ([Euc $]_{\text {Tot }}$ ). The 
133 liposomal suspension underwent ultracentrifugation (Optima ${ }^{\mathrm{TM}}$ Ultracentrifuge, Beckman

134 Coulter, USA) at $170000 \mathrm{~g}$ for $1 \mathrm{~h}$ at $4{ }^{\circ} \mathrm{C}$. Aliquots were removed from the supernatant to

135 determine the concentration of free Euc $\left([\mathrm{Euc}]_{\mathrm{F}}\right)$. The EE of Euc was calculated as

136 follows:

$$
E E(\%)=\frac{[\text { Euc }]_{T o t}-[\text { Euc }]_{F}}{[\text { Euc }]_{T o t}} \times 100(2)
$$

137 where $[\mathrm{Euc}]_{\mathrm{Tot}}$ and $[\mathrm{Euc}]_{\mathrm{F}}$ corresponds to the concentration of total and free Euc,

138 respectively.

139 The LR of Euc was calculated by the following equation:

$$
L R=\frac{m_{\text {liposomal suspension }}-m_{\text {filtrate }}}{m_{\text {Initial }}} \times 100 \text { (3) }
$$

142 The LR after freeze-drying was calculated using the following equation:

$$
L R_{f d}=\frac{m_{\text {reconstituted liposomes }}}{m_{\text {Initial }}} \times 100
$$

143 where $\mathrm{m}_{\text {Initial }}$ for Euc-DCL is the initial mass of Euc used to prepare CD/Euc inclusion

144 complex. For Euc-CL, $\mathrm{m}_{\text {initial }}$ is the initial mass of Euc added to the organic phase during

145 liposome preparation. $\mathrm{m}_{\text {reconstituted liposomes }}$ is the mass of Euc totally found in the liposomal 146 suspension after freeze-drying and reconstitution of liposomes.

\section{$\begin{array}{lll}147 & 2.7 & \text { HPLC method }\end{array}$}

148 The concentration of Euc was determined by HPLC. Stock standard solutions of Euc (1 $149 \mathrm{mg} / \mathrm{mL})$ and of the internal standard, eugenol $(1 \mathrm{mg} / \mathrm{mL})$ were prepared in methanol. 150 Aliquots were removed from the Euc stock solution and diluted in methanol to obtain 151 final concentrations of Euc ranging from 100 to $1000 \mu \mathrm{g} / \mathrm{mL}$. The diluted solution of 152 eugenol $(1 \mu \mathrm{g} / \mathrm{mL})$ was prepared in methanol. Two hundred $\mu \mathrm{L}$ of each filtrate were 
153 added to $200 \mu \mathrm{L}$ of eugenol $(1 \mu \mathrm{g} / \mathrm{mL})$ and $400 \mu \mathrm{L}$ of methanol. The samples were

154 sonicated for $10 \mathrm{~min}$ at room temperature. The samples were analyzed by HPLC (Agilent

155 Technologie 1200 series) using an analytical column C18 $15 \mathrm{~cm}$ x $4.6 \mathrm{~mm}, 5 \mu \mathrm{m}$,

156 (Agilent Technologies). The mobile phase was a mixture of methanol and water (70/30).

157 The flow rate was fixed at $1 \mathrm{~mL} / \mathrm{min}$ and the detection was set at $206 \mathrm{~nm}$.

158 The retention times of eugenol and Euc were 4.3 and 7.3 min, respectively.

$159 \quad 2.8 \quad$ Fluorescence anisotropy measurements

160 The effect of Euc, HP-ß-CD and freeze-drying process on the membrane fluidity of 161 vesicles (blank-CL, blank-DCL, Euc-CL, Euc-DCL), the steady state fluorescence 162 polarization measurements were determined using a Cary Eclipse Fluorescence 163 Spectrophotometer (Agilent Technologies). DPH was dissolved in tetrahydrofuran (4.30 $16410^{-3} \mathrm{~mol} / \mathrm{L}$ ) then an aliquot was added to the vesicles suspension to obtain

165 DPH:phospholipid molar ratio of 1:500. The measurements were conducted following the 166 protocol described previously ${ }^{[23]}$.

\section{$167 \quad 2.9 \quad$ Release of Euc}

168 Multiple headspace extraction (MHE) coupled to gas chromatography was used to study

169 the release of Euc form freeze-dried CL and DCL as well as from reconstituted vesicles

170 after 6 months of storage at $4^{\circ} \mathrm{C}$. Freeze-dried powders of Euc-CL, Euc-DCL (10 mg),

171 reconstituted Euc-CL, Euc-DCL in $10 \mathrm{~mL}$ water, inclusion complex solution prepared at

172 a molar ratio HP-ß-CD/Euc of 5:1, and Euc dissolved in water $(0.2 \mathrm{mg} / \mathrm{mL})$ were placed

173 in $22 \mathrm{ml}$ sealed vials. The protocol described in a previous study ${ }^{[13]}$ was used.

174 After equilibrium, the release of Euc at time t was calculated as follows:

175

$$
\text { Percentage of remaining Euc }=\frac{A_{t}}{A_{1}} \times 100 \text { (6) }
$$


176 Where, $A_{t}$ and $A_{1}$ corresponds to the area of the chromatographic peak of Euc at time $t$

177 and at the first extraction, respectively. The plot of $\ln \left(\mathrm{A}_{t} / \mathrm{A}_{1}\right)$ as a function of time

178 followed a first-order release kinetics. The release rate constant $\mathrm{K}$ was calculated using

179 the following equation:

$$
\operatorname{Ln}\left(\mathrm{A}_{\mathrm{t}} / \mathrm{A}_{1}\right)=-\mathrm{Kt}+\mathrm{b} \quad(7)
$$

\section{$181 \quad 2.10$ Loading capacity determination}

182 The amount of Euc in freeze-dried forms of CL and DCL was determined after 6 months

183 of storage at $4^{\circ} \mathrm{C}$ using $\mathrm{MHE}$. The concentration of Euc was determined according to the 184 protocol developed by Kolb \& Ettre ${ }^{[24]}$ and used in our previous studies ${ }^{[13]}$.

185 Loading capacity (LC) of Euc is expressed as mg of encapsulated Euc per gram of 186 liposomes.

$$
L C=\frac{E u c_{\text {exp }}(\mathrm{mg})}{\text { masse of liposomes }(m g)}(8)
$$

\section{$188 \quad 2.11$ Statistical analysis}

189 Statistical analysis was performed using the Student's t-test. $P$ values equal or less than 1900.05 were considered statistically significant.

\section{$191 \quad 3 \quad$ Results and discussion}

\section{3.1 Determination of the optimal HP-B-CD/Euc inclusion complex}

193 Table 1 presents the encapsulation efficiency of Euc using various HP- $\beta$-CD

194 concentrations. $\mathrm{EE}_{\mathrm{CD}}$ values were calculated using equation 1. It was found that a HP- $\beta$ -

195 CD concentration of $50 \mathrm{mM}$ was necessary to dissolve the total amount of Euc. Same

196 result was found for anethole, another essential oil component ${ }^{[12]}$. HP- $\beta-C D$ :Euc of 5:1

197 molar ratio was used subsequently to prepare DCL formulations. 


\subsection{Liposomes characterization before and after freeze-drying}

\section{$199 \quad$ 3.2.1 Size, polydispersity index and zeta potential values}

200 CL and DCL liposomal suspension were prepared by the ethanol injection method using 201 hydrogenated phospholipids (Phospholipon 90H) and cholesterol. Table 2 summarizes

202 the size, polydispersity index and zeta potential values of vesicles before and after freeze203 drying.

204 Before freeze-drying, the size of blank-DCL, Euc-CL and Euc-DCL showed no 205 significant difference compared to blank-CL, since similar range of size (179 to $201 \mathrm{~nm}$ )

206 was obtained. These results demonstrated that the presence of HP-ß3-CD and/or Euc did 207 not affect the liposome particle size. Same results were reported for encapsulation of 208 anethole $^{[11,12]}$, estragole ${ }^{[13]}$ and clove essential oil ${ }^{[14]}$ in liposomes composed of 209 hydrogenated phospholipids. HP- $\beta-C D$ and essential oil components are able to affect the

210 size of liposomes composed of unsaturated phospholipids ${ }^{[25]}$. Besides, polydispersity

211 index values for all batches were less than 0.2, suggesting that liposomes suspensions

212 were homogenous (Table 2). The zeta potential values for the various formulations were

213 negative ranging between ( -2 to $-9 \mathrm{mV}$ ) suggesting that the presence of HP-ß-CD or Euc

214 did not affect the zeta potential values.

215 In a previous work, we optimized the freeze-drying of hydrogenated liposomes using HP-

216 B-CD as cryoprotectant for CL and DCLs ${ }^{[12]}$. The results showed that dispersing the

217 pellet of CL in HP-ß-CD solution (25 or $50 \mathrm{mM}$ ), that of DCL in water or in HP-B-CD

218 solution (10 to $100 \mathrm{mM}$ ) protect the structures during freeze-drying. For DCL pellet, HP-

$219 \quad 3-\mathrm{CD}$ solution was used at the same CD concentration present in the internal aqueous 220 phase of vesicles. The Euc-DCL formulation was prepared using HP-B-CD/Euc molar 
221 ratio of $5: 1$ where $\mathrm{HP}-\beta-\mathrm{CD}(50 \mathrm{mM})$ and Euc $(10 \mathrm{mM})$ were used to prepare the

222 inclusion complex. Thus HP-ß-CD (50 mM) was used as a cryoprotector for DCL

223 formulations.

224 After freeze-drying, the size, pdI and zeta potential values were maintained proving that

225 the freeze-drying process was well optimized and could be used for a large number of

226 bioactive compounds loaded CL or DCL (Table 2).

\section{$227 \quad$ 3.2.2 Morphology}

228 The TEM images of phospholipon $90 \mathrm{H}$ liposomes obtained before and after freeze-drying 229 showed the formation of nanometer-sized vesicles. Figure 1 (A-B) and (C-D) presented 230 the images of blank-DCL and Euc-DCL obtained before and after freeze-drying,

231 respectively. The vesicles appeared to be oligolamellar and spherical in shape. Similar 232 morphology was found for DCLs loading anethole ${ }^{[12,26]}$, estragole ${ }^{[13]}$ and eugenol ${ }^{[14]}$.

233 The sizes of the vesicles were well below those determined by DLS technique. In this

234 work, the number of TEM images taken for each formulation was not enough to 235 determine the mean particle size for each batch; moreover some factors may affect the 236 results obtained by DLS such as temperature and the viscosity of the sample.

\section{$237 \quad 3.2 .3 \quad$ Encapsulation efficiency}

238 The encapsulation efficiency (EE) and the loading rate (LR) of Euc-loaded liposomes

239 (CL and DCL) before and after freeze-drying were calculated using equations (2-4) and

240 the values are listed in Table 3. The concentration of Euc before freeze-drying in Euc-CL

241 was not detected by HPLC analysis. Same results were obtained for estragole ${ }^{[13]}$. The

242 Henry's law constant, previously determined by static headspace-gas chromatography at

$24330^{\circ} \mathrm{C}$ were of 0.01 and 0.03 for Euc and estragole, respectively. It has been reported that 
244 these molecules exhibited higher volatility compared to other essential oil compounds;

245 the Henry's law constant for isoeugenol, pulegone, terpineol, and thymol at $30^{\circ} \mathrm{C}$ were of

$2460.005,0.005,0.001$ and 0.003 respectively. The Henry's law constant was increased to

$2470.05,0.07,0.008,0.009,0.003$ and 0.009 for Euc, estargole, isoeugenol, pulegone,

248 terpineol, and thymol respectively upon increasing the temperature from 30 to $60^{\circ} \mathrm{C}^{[25]}$.

249 The chromatogram obtained for eucalyptol at $60^{\circ} \mathrm{C}$ did not shown any additional peak or

250 presence of a degradation product during the time of the analysis $(8 \mathrm{~min})$. The

251 volatilization of Euc during the preparation of liposomes at $55^{\circ} \mathrm{C}$ can explain the dramatic

252 loss of this volatile agent.

253 The Euc-DCL system improved the encapsulation of Euc with EE value $78.24 \pm 2.33 \%$,

254 and the LR value was $8.59 \pm 1.61 \%$. Hence, DCL improved the encapsulation of different

255 drugs including volatile ones like trans-anethole ${ }^{[12]}$, estragole ${ }^{[13]}$. Some non-volatile

256 compounds like betamethasone ${ }^{[27]}$, celcoxib ${ }^{[28]}$ and curcumin ${ }^{[29]}$ were also better

257 encapsulated in DCL compared to conventional liposomes.

258 The LR of Euc significantly decreased after freeze-drying to $0.81 \pm 0.22$ for Euc-DCL

259 (Table 3). Also, a remarkable anethole loss from DCL was obtained during freeze-drying

260 process ${ }^{[12]}$. The vacuum pressure of $100 \mu$ bar provokes probably anethole and Euc loss.

261 For that, further experiments should be performed to optimize the freeze-drying of DCL

262 loading highly volatile compounds.

\section{$263 \quad 3.2 .4 \quad$ Fluorescence anisotropy}

264 Table 4 showed the DPH anisotropy values obtained at 25 and $37^{\circ} \mathrm{C}$ for blank-CL, blank-

265 DCL, Euc-CL and Euc-DCL before and after freeze-drying. 
266 The membrane fluidity increased with the temperature in accordance with literature ${ }^{[23]}$.

267 Compared to blank-CL, the presence of Euc or the inclusion complex HP- $\beta-C D / E u c$ did

268 not affect the DPH anisotropy values at 25 and $37^{\circ} \mathrm{C}$. The result obtained for Euc-CL has

269 been expected since Euc was not detected in Euc-loaded CL by HPLC. Gharib, et al ${ }^{\text {[30] }}$

270 demonstrated that the presence of monoterpenes (pulegone, terpineol and eucalyptol) at

271 high concentration of monoterpene to phospholipid molar ratios (10\%) decreased in a

272 concentration dependent manner the anisotropy value of DPPC membrane at 28,41 and

$27350{ }^{\circ} \mathrm{C}$. Besides, the presence of HP- $\beta-\mathrm{CD}$ as cryoprotectant did not affect the membrane

274 fluidity, since no changes of the DPH anisotropy values were observed before and after

275 freeze-drying for all batches (Table 4).

\section{$276 \quad 3.3 \quad$ Release studies}

277 The Euc release experiments from freeze-dried HP-ß-CD/Euc inclusion complex, Euc-CL

278 and Euc-DCL were conducted at $60^{\circ} \mathrm{C}$ using the MHE method after 6 months of storage

279 at $4^{\circ} \mathrm{C}$. Figure 2 showed the Euc peak areas of the external standard $(0.2 \mathrm{ppm}$ of Euc in

280 water), inclusion complex HP-ß-CD/Euc, Euc-CL and Euc-DCL (in powder or

281 reconstituted in water). The amount of Euc in powders (CL and DCL) was very low

282 compared to reconstituted liposomes suggesting the high retention of Euc in powder

283 samples. Thus, the powders were dissolved in $10 \mathrm{~mL}$ water and placed in sealed vials and

284 analyzed by MHE. Compared to CL, the encapsulation of Euc was better in DCL, while

285 the inclusion complex showed the higher ability to retain Euc (Figure 2). Reconstituted

286 batches were then considered for the release study and for the determination of the LC

$287 \mathrm{~m}_{\text {Euc }} / \mathrm{m}_{\text {powder }}$ of Euc.

288 The remaining percentage of Euc as free drug or when encapsulated in lipid vesicles are 
$290(58.5 \%)$ than in free Euc (78.2\%). Thus, CL prepared in our conditions failed to be an

291 effective delivery system for Euc. For the other formulations, the remaining percentage of

292 Euc increased in the order: free Euc $(78.2 \%)>$ Euc-DCL $(82.5 \%)>$ HP-ß-CD:Euc

293 (85\%). Thus the Euc of eucalyptol in DCL system and HP-ß-CD:Euc inclusion complex

294 improve its release compared to free Euc.

295 The release rate constant was calculated according to equation 7 and the values are listed

296 in Table 5. The retention of Euc was improved by 1.6 and 1.3 times for HP-ß-CD/Euc

297 inclusion complex and Euc-DCL compared to free Euc (0.2 ppm).

\section{$298 \quad 3.4$ Determination of loading capacity of Euc}

299 The LC values of Euc in HP-B-CD/Euc inclusion complex, Euc-CL and Euc-DCL, after 6

300 months of storage at $4^{\circ} \mathrm{C}$, were determined using equation 8 and presented in Table 6 . As

301 expected, a small amount of Euc was obtained in Euc-CL with LC value $0.0155 \mu \mathrm{g}$ of

302 Euc/mg of powder, since Euc was not detected by HPLC method. However, Hammoud et

303 al $^{[25]}$ studied the encapsulation of Euc in liposomes composed of unsaturated soybean 304 phospholipid (Lipoid S100) and the results showed that CL loading Euc had a low 305 loading capacity value of Euc (1.1 $\mu \mathrm{g}$ of drug/mg of total organic components). The 306 encapsulation of Euc in DCL system was 38 times greater than CL formulations with LC 307 values of $0.6 \mu \mathrm{g}$ of Euc/mg of powder (Table 6). However, the higher LC value of Euc 308 was obtained with HP-ß-CD/Euc inclusion complex with $4.5 \mu \mathrm{g}$ of Euc/mg of powder.

309 These results suggest that DCL system improve the encapsulation of Euc compared to

310 CL. The inclusion complex HP- $\beta-C D /$ Euc showed the best ability to retain high amount 311 of Euc. 


\section{Conclusion}

313 In this paper, we demonstrated a successful freeze-drying of Euc loaded Phospholipon

$31490 \mathrm{H}$ liposomes where the characteristics of hydrogenated-liposomes (size, pdI, zeta

315 potential and morphology) were maintained after freeze-drying using HP-B-CD as

316 cryoprotectant for CL and DCL systems. HP-ß-CD/Euc and Euc-DCL controlled drug

317 release and displayed a good stability after 6 months of storage at $4{ }^{\circ} \mathrm{C}$ in powder form.

318 These results revealed that freeze-dried CD/drug inclusion complex and DCL reduced the

319 volatilization of Euc and improved its retention. This work suggests that the encapsulated

320 Euc could be considered as a suitable approach for the insecticide application.

321 Conflicts of interest

322 We confirm that there are no known conflicts of interest associated with this publication.

\section{Acknowledgements}

324 The research was supported by the Research Funding Program at the Lebanese University 325 (2018-2020) and the "Agence Universitaire de la Francophonie", projet PCSI (2018326 2020). 


\section{References}

[1]. U. R. Juergens, Drug Res. 2014, 64, 638.

[2]. D. R. Batish, H. P. Singh, N. Setia, S. Kaur, R. K. Kohli, Z. Naturforsch C. J. Biosci. 2006, 61, 465.

[3]. R, Scriven, C. E. Meloan, Ohio J. Sci. 1984, 84, 85.

[4].S. A. Corbet, G. W. Danahar, V. King, C. L. Chalmers, C. F. Tiley, Entom. Exp. App. 1995, 75, 229.

[5].J. Mediouni Ben Jemâa, S. Haouel, M. Bouaziz, M. L. Khouja, J. Stored Prod. Res. 2012, 48, 61.

[6].A. K. Dhakad, V. V. Pandey, S. Beg, J. M. Rawat, A. Singh, J. Sci. Food Agric. 2018, 98, 833 .

[7].P. R. Lima, T. S. de Melo, K. M. Carvalho, I. B. de Oliveira, B. R. Arruda, G. A. de Castro Brito, V. S. Rao, F. A. Santos, Life Sci. 2013, 92, 1195.

[8].C. Turek, F. C. Stintzing, Comp. Rev. Food Sci. Food Safety 2013, 12, 40.

[9].R. Azerard, ChemPlusChem. 2014, doi.org/10.1002/cplu.201300422 .

[10]. B. McCormack, G. Gregoriadis, Int. J. Pharm. 1994, 112, 249.

[11]. R. Gharib, L. Auezova, C. Charcosset, H. Greige-Gerges, Food Chem. 2017, 218,365

[12]. R. Gharib, H. Greige-Gerges, S. Fourmentin, C. Charcosset, Food Chem. 2018, $267,67$.

[13]. R. Gharib, S. Haydar, C. Charcosset, S. Fourmentin, H. Greige-Gerges, J. Drug Del. Sci. Tech. 2019, 52, 794. 
[14]. C. Sebaaly, C. Charcosset, S. Stainmesse, H. Fessi, H. Greige-gerges, Carbohydr. Polym. 2016, 138, 75.

[15]. J. Azzi, A. Jraij, L. Auezova, S. Fourmentin, H. Greige-Gerges, Food Hydrocoll. 2018, 81, 328.

[16]. J Azzi, L. Auezova, P. E. Danjou, S. Fourmentin, H. Greige-Gerges, Food Chem. 2018, 255, 399.

[17]. J. Szejtli, Pure Appl. Chem. 2004, 76, 1825.

[18]. M. Kfoury, L. Auezova, S. Fourmentin, H. Greige-Gerges, J. Incl. Phenom. Macro. Chem. 2014, 80, 51.

[19]. H. Anwekar, S. Patel, A. K. Singhai, Int. J. Pharm. Life Sci. 2011, 2, 945.

[20]. C. Chen, D. Han, C. Cai, X. Tang, J. Control. Release 2010, 142, 299.

[21]. A. Wieber, T. Selzer, J. Kreuter, Europ. J. Pharm. Biopharm. 2012, 80, 358.

[22]. M. M. Domingues, P. S. Santiago, M. A. Castanho, N. C. Santos, J Pept Sci. 2008, 14, 394.

[23]. R. Abboud, C. Charcosset, H. Greige-Gerges, J. Membr. Biol. 2016, 249, 327.

[24]. B. Kolb, L. S. Ettre, Static Headspace-Gas Chromatography: Theory andPractice (Second edition), New Jersey: John Wiley \& Sons, Inc. Hoboken 2006

[25]. Z. Hammoud, R. Gharib, S. Fourmentin, A. Elaissari, H. Greige-Gerges, Int. J. Pharm. 2019, 561, 1610.

[26]. R. Gharib, H. Greige-Gerges, A. Jraij, L. Auezova, C. Charcosset, Carbohydr. Polym. 2016, 154, 276. 
[27]. G. Piel, M. Piette, V. Barillaro, D. Castagne, B. Evrard, L. Delattre, Int. J. Pharm. 2006, 312, 75.

[28]. S. K. Jain, Y. Gupta, A. Jain, M, Bhola, Drug Deliv. 2007, 14, 327.

[29]. S. S. Dhule, P. Penfornis, T. Frazier, R. Walker, J. Feldman, G. Tan, J. He, A. Alb, V. John, R. Pochampally, Nanomedicine 2012, 8, 440.

[30]. R. Gharib, L. Auezova, C. Charcosset, H. Greige- Gerges, J. Iran. Chem. Soc. $2018,15,75$. 


\section{Figures}
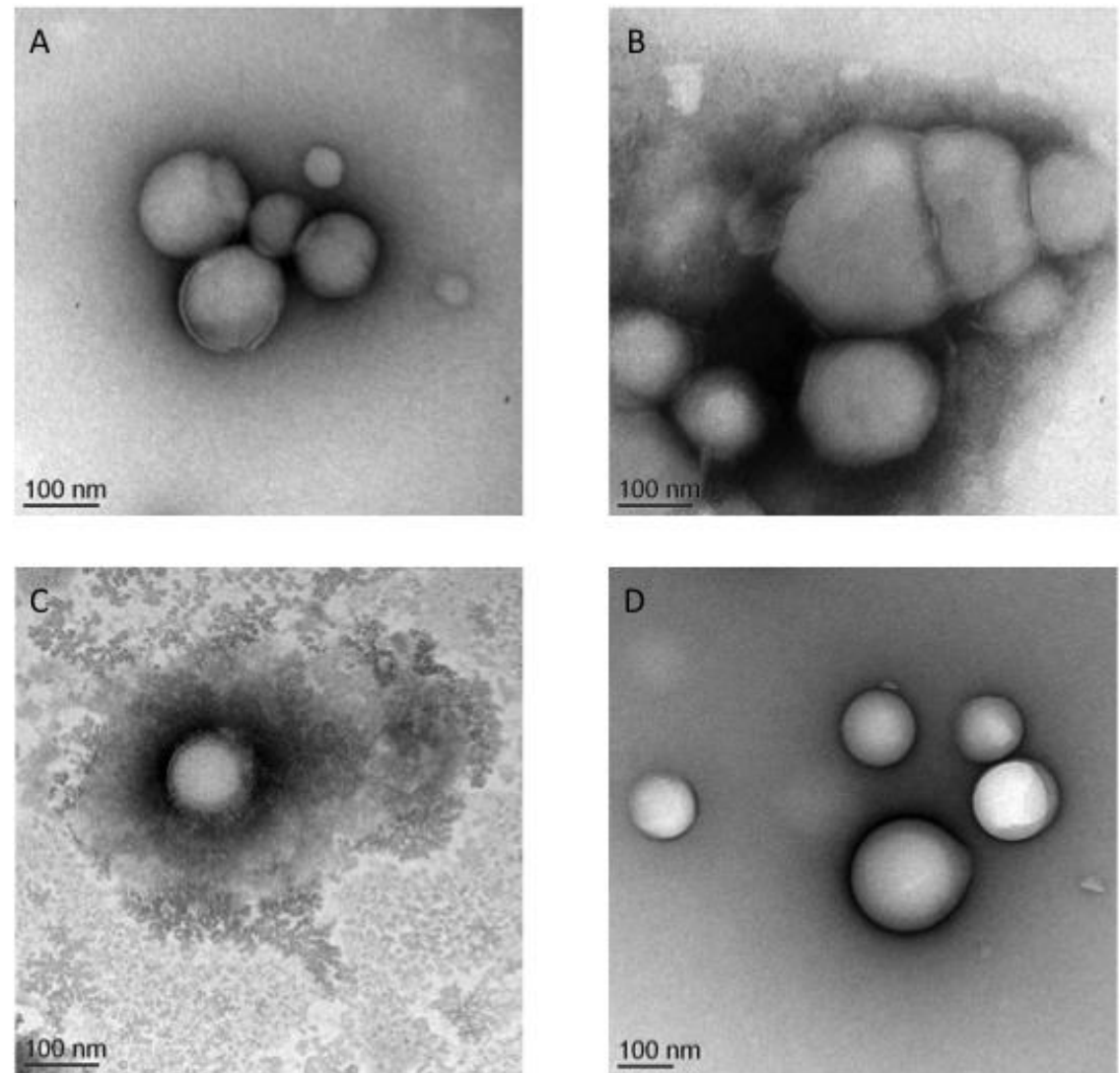

Figure 1: TEM images of Phospholipon 90H liposomes before freeze-drying: blank-DCL 50 (A) and Euc-DCL 50 (B) and after freeze-drying: blank-DCL 50 (C); and Euc-DCL 50 (D). 


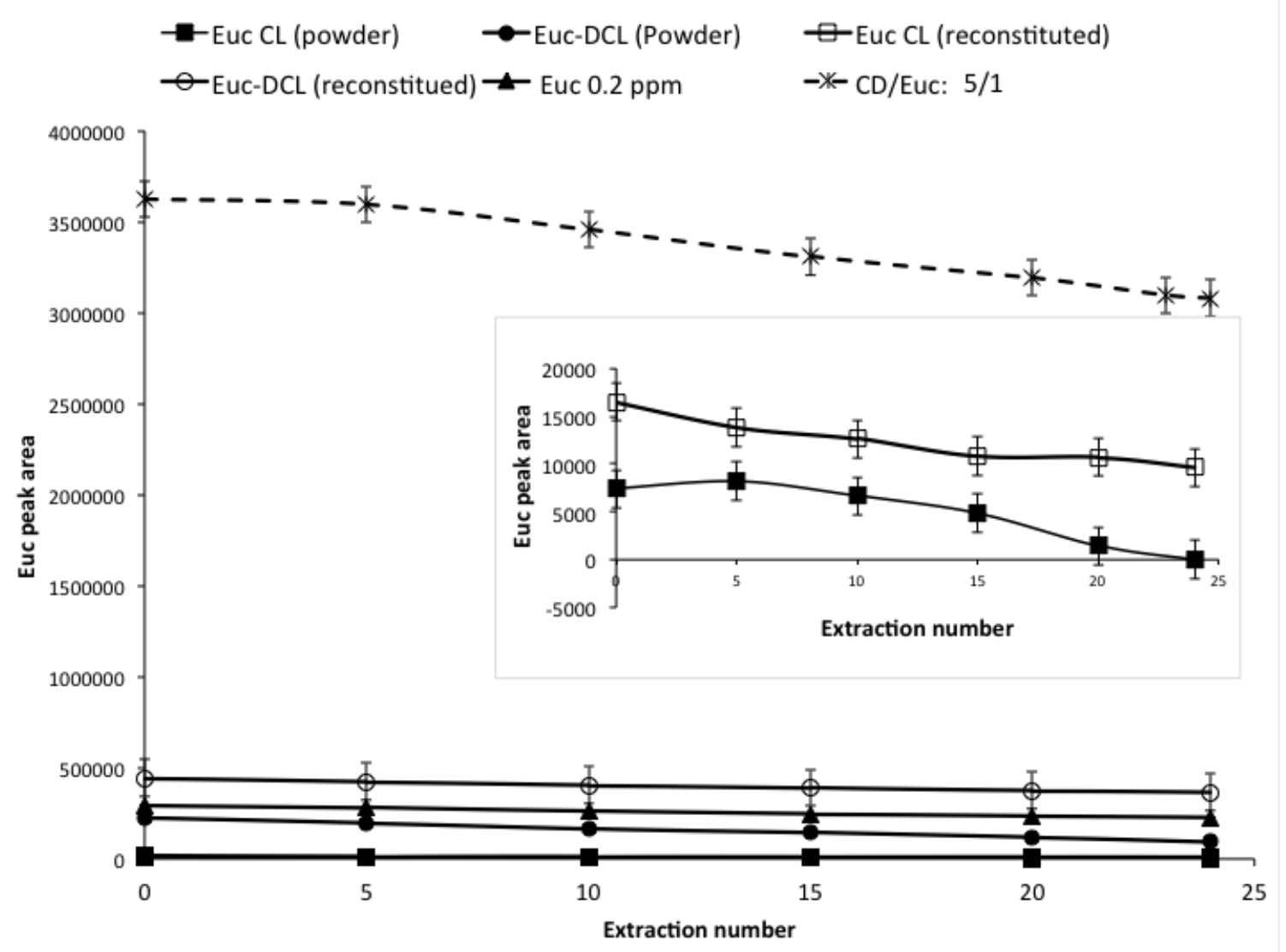

Figure 2: The Euc peak areas of the external standard ( $0.2 \mathrm{ppm}$ of Euc in water), inclusion complex HP-ß-CD:Euc (5:1 molar ratio in water) and Euc-CL and Euc-DCL (in powder or reconstituted in water). The insert represents the peak areas for CL in powder form and reconstituted in water. 


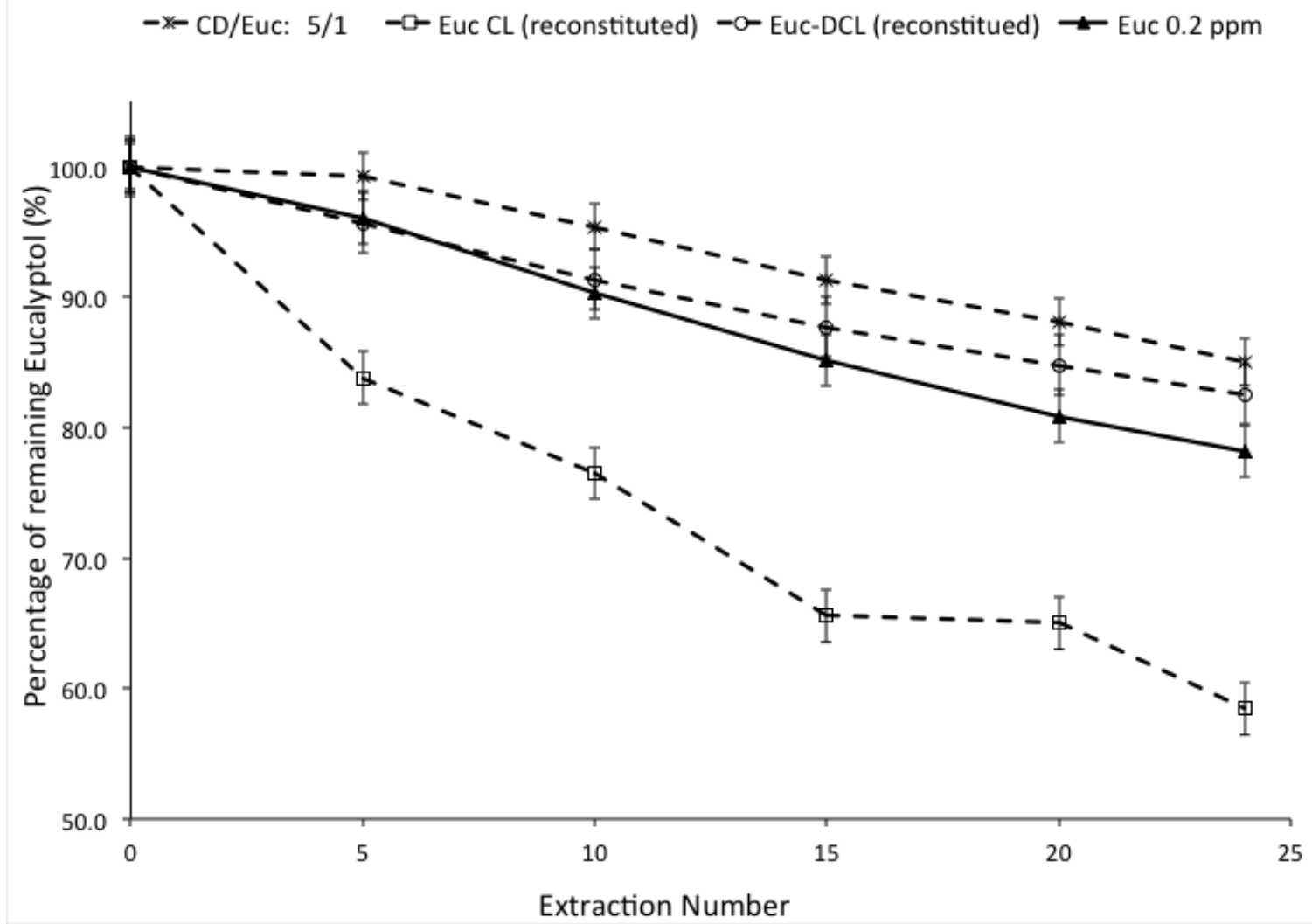

Figure 3: The percentage of remaining Euc from Euc external standard $(0.2 \mathrm{ppm}$ of eucalyptol in water), HP-ß-CD:Euc inclusion complex and Euc-CL and DCL (reconstituted). 
Table 1: The encapsulation efficiency of Euc in HP-ß-CD/Euc inclusion complex using different HP-ß3-CD concentrations.

\begin{tabular}{|c|c|}
\hline [HP-B-CD] $\mathbf{~ m M}$ & $\mathbf{E E}_{\mathbf{C D}} \mathbf{( \% )}$ \\
\hline 0 & $72.72 \pm 10.16$ \\
\hline 10 & $71.51 \pm 6.95$ \\
\hline 25 & $92.78 \pm 5.37$ \\
\hline 50 & $98.66 \pm 6.20$ \\
\hline 100 & $99.08 \pm 2.2$ \\
\hline
\end{tabular}

Table 2: Size, polydispersity index, zeta potential values for fresh and reconstituted Euc-

CL and Euc-DCL from Phospholipon 90H before and after freeze-drying.

The values obtained after lyophilization were compared to those before lyophilization.

\begin{tabular}{|c|c|c|c|c|c|c|c|}
\hline & \multicolumn{3}{|c|}{ Before freeze-drying } & & \multicolumn{3}{|c|}{ After freeze-drying } \\
\hline & Size $(\mathrm{nm})$ & pdI & Zeta $(\mathrm{mV})$ & $\begin{array}{l}\text { Dispersing of } \\
\text { the pellet with }\end{array}$ & $\begin{array}{l}\text { Size } \\
(\mathrm{nm})\end{array}$ & pdI & Zeta $(\mathrm{mV})$ \\
\hline \multirow{2}{*}{ Blank-CL } & \multirow{2}{*}{$179 \pm 9$} & \multirow{2}{*}{$0.08 \pm 0.01$} & \multirow{2}{*}{$-9.1 \pm 6.3$} & CD $25 \mathrm{mM}$ & $169 \pm 8$ & $0.22 \pm 0.04$ & $-7.4 \pm 1.7$ \\
\hline & & & & CD $50 \mathrm{mM}$ & $180 \pm 17$ & $0.15 \pm 0.02 *$ & $-5.0 \pm 4.0^{*}$ \\
\hline \multirow{2}{*}{ Euc-CL } & \multirow{2}{*}{$180 \pm 4$} & \multirow{2}{*}{$0.09 \pm 0.02$} & \multirow{2}{*}{$-8.8 \pm 3.5$} & CD $25 \mathrm{mM}$ & $182 \pm 9$ & $0.22 \pm 0.08$ & $-14.8 \pm 4.5^{*}$ \\
\hline & & & & CD $50 \mathrm{mM}$ & $175 \pm 3$ & $0.15 \pm 0.04$ & $-8.9 \pm 2.1$ \\
\hline Blank-DCL & $196 \pm 2$ & $0.17 \pm 0.01$ & $-2.4 \pm 2.5$ & CD $50 \mathrm{mM}$ & $193 \pm 4$ & $0.14 \pm 0.03$ & $-14.0 \pm 1.7$ \\
\hline Euc-DCL & $201 \pm 9$ & $0.20 \pm 0.00$ & $-8.8 \pm 5.6$ & CD $50 \mathrm{mM}$ & $208 \pm 21$ & $0.21 \pm 0.03$ & $-10.1 \pm 5.3$ \\
\hline
\end{tabular}


Table 3: Encapsulation efficiency of Euc for fresh CL and DCL suspensions and loading rate of Euc in fresh and reconstituted CL and DCL before and after freeze-drying

\begin{tabular}{|l|c|l|c|}
\hline & \multicolumn{2}{|c|}{$\begin{array}{c}\text { Before freeze-drying } \\
\text { Fresh liposomes }\end{array}$} & $\begin{array}{c}\text { After freeze-drying } \\
\text { Reconstituted liposomes }\end{array}$ \\
\hline & EE (\%) & LR $_{\mathbf{I}}(\boldsymbol{\%})$ & LR $_{\text {fd }}(\%)$ \\
\hline Euc-CL & ND & ND & ND \\
\hline Euc-DCL 50 & $78.24 \pm 2.33$ & $8.59 \pm 1.61$ & $0.81 \pm 0.22 *$ \\
\hline
\end{tabular}

ND: not detected

- indicates a value of $\mathrm{P}<0.05$ statistically significant.

Table 4: DPH fluorescence anisotropy measurements for blank-CL, blank-DCL, Euc-CL and Euc-DCL made from Phospholipon $90 \mathrm{H}$ and cholesterol at 25 and $37^{\circ} \mathrm{C}$ before and after freeze-drying.

\begin{tabular}{|l|c|c|c|c|}
\hline & \multicolumn{2}{|c|}{ Before lyophilization } & \multicolumn{2}{c|}{ After lyophilization } \\
\hline & $\mathbf{2 5}^{\circ} \mathbf{C}$ & $\mathbf{3 7}^{\mathbf{}} \mathbf{C}$ & $\mathbf{2 5}^{\mathbf{C}}$ & $\mathbf{3 7}^{\mathbf{C}} \mathbf{C}$ \\
\hline Blank-CL & $0.22 \pm 0.0$ & $0.17 \pm 0.0$ & n.d. & n.d. \\
\hline Blank DCL & $0.21 \pm 0.0$ & $0.17 \pm 0.0$ & $0.22 \pm 0.00$ & $0.17 \pm 0$ \\
\hline Euc-CL & $0.22 \pm 0.0$ & $0.17 \pm 0.0$ & n.d. & n.d. \\
\hline Euc-DCL & $0.21 \pm 0.0$ & $0.18 \pm 0.0$ & $0.22 \pm 0.01$ & $0.17 \pm 0$ \\
\hline
\end{tabular}

Values are expressed as the means of three repetitions \pm SD; n.d.: not determined $\mathrm{P}<0.05$ compared to those before freeze-drying 
Table 5: Release rate constants of free Euc, HP-ß-CD/Euc inclusion complex, Euc-CL and Euc-DCL formulations

\begin{tabular}{|l|c|}
\hline & $\begin{array}{c}\text { Release rate constant } \mathbf{K} \\
\left(\mathbf{m i n}^{-\mathbf{1}}\right)\end{array}$ \\
\hline Euc $0.2 \mathrm{ppm}$ & 0.011 \\
\hline Euc-CL reconstituted & 0.021 \\
\hline Euc-DCL reconstituted & 0.008 \\
\hline HP-ß-CD:Euc complex & 0.007 \\
\hline
\end{tabular}

Table 6: Loading capacity of Euc in HP-ß-CD/Euc inclusion complex and in Euc-CL and Euc-DCL carrier systems after 6 months of storage at $4^{\circ} \mathrm{C}$.

\begin{tabular}{|c|c|}
\hline & LC ( $\boldsymbol{\mu g}$ of Euc/mg of powder) \\
\hline HP-ß-CD:Euc & 4.5 \\
\hline Euc-CL & 0.0155 \\
\hline Euc-DCL & 0.6 \\
\hline
\end{tabular}

